University of Wollongong

Research Online

Australian Institute for Innovative Materials -

Papers

Australian Institute for Innovative Materials

$1-1-2012$

\title{
Large scale synthesis of V-shaped rutile twinned nanorods
}

Weigang Lu

Baylor University

Britain Bruner

Baylor University

Gilberto Casillas

University of Texas at San Antonio, gilberto@uow.edu.au

Jibao $\mathrm{He}$

Tulane University

Miguel Jose-Yacaman

University of Texas at San Antonio

See next page for additional authors

Follow this and additional works at: https://ro.uow.edu.au/aiimpapers

Part of the Engineering Commons, and the Physical Sciences and Mathematics Commons

Research Online is the open access institutional repository for the University of Wollongong. For further information contact the UOW Library: research-pubs@uow.edu.au 


\title{
Large scale synthesis of V-shaped rutile twinned nanorods
}

\author{
Abstract \\ V-shaped rutile twinned nanorods (VRTNs) with different aspect ratios were synthesized on large scale \\ using a one-pot organic solvothermal method. Although several different types of VRTNs were produced, \\ (101) twinned nanorods were the major product with minor amounts of (301) twins and straight \\ nanorods. STEM, TEM and XRD characterizations show that [001] is the growth direction of VRTNs, with \\ surfaces dominated by $\{110\}$ facets. Intermediate formation of both brookite and anatase nanorods \\ provides dual pathways to VRTNs' growth: one path in which anatase seeds trigger and promote brookite \\ nanorod growth; and a second path in which anatase nanorods are formed by oriented attachment. \\ Subsequent dissolution of both brookite and anatase nanorods provides titania to support VRTNs growth. \\ Keywords \\ twinned, rutile, shaped, v, synthesis, nanorods, scale, large \\ Disciplines \\ Engineering | Physical Sciences and Mathematics \\ Publication Details \\ Lu, W., Bruner, B., Casillas, G., He, J., Jose-Yacaman, M. \& Farmer, P. J. (2012). Large scale synthesis of V- \\ shaped rutile twinned nanorods. CrystEngComm, 14 (9), 3120-3124.

\section{Authors} \\ Weigang Lu, Britain Bruner, Gilberto Casillas, Jibao He, Miguel Jose-Yacaman, and Patrick J. Farmer
}




\title{
CrystEngComm
}

\section{Large scale synthesis of $\mathbf{V}$-shaped rutile twinned nanorods $\dagger$}

\author{
Weigang Lu, ${ }^{* a}$ Britain Bruner, ${ }^{a}$ Gilberto Casillas, ${ }^{b}$ Jibao He, ${ }^{c}$ Miguel Jose-Yacaman ${ }^{b}$ and Patrick J. Farmer ${ }^{* a}$ \\ Received 22nd November 2011, Accepted 17th January 2012 \\ DOI: 10.1039/c2ce06564a
}

\begin{abstract}
V-shaped rutile twinned nanorods (VRTNs) with different aspect ratios were synthesized on large scale using a one-pot organic solvothermal method. Although several different types of VRTNs were produced, (101) twinned nanorods were the major product with minor amounts of (301) twins and straight nanorods. STEM, TEM and XRD characterizations show that [001] is the growth direction of VRTNs, with surfaces dominated by $\{110\}$ facets. Intermediate formation of both brookite and anatase nanorods provides dual pathways to VRTNs' growth: one path in which anatase seeds trigger and promote brookite nanorod growth; and a second path in which anatase nanorods are formed by oriented attachment. Subsequent dissolution of both brookite and anatase nanorods provides titania to support VRTNs growth.
\end{abstract}

\section{Introduction}

Titania, $\mathrm{TiO}_{2}$, is a widely used material, with diverse applications such as in paints, solar cells, and environmental remediation technologies. ${ }^{1-3}$ It is also often used as a model metal oxide to address fundamental scientific issues such as interfacial electron transfer and defect effects in catalysis. ${ }^{4,5}$ Among the three common titania polymorphs (rutile, anatase, and brookite), anatase has the highest catalytic activity; atomic level catalysis studies are typically conducted on a rutile $\mathrm{TiO}_{2}(110)$ surface due to its excellent electronic properties and well understood surface chemistry. ${ }^{4,5}$ Rutile is the preferred polymorph because it has the lowest bulk Gibbs free energy and lowest molecular volume; ${ }^{6}$ it has a prototypic $\mathrm{C} 4$ crystallographic structure (space group $P 42 / \mathrm{mnm}$ ), at times displaying well defined elongated prismatic shapes with twinning in the form of elbows and hearts, as well as reticulated twins. ${ }^{7}$ The higher energy phase anatase and brookite will convert to rutile when heated. The transition of anatase to rutile has been observed under many synthetic conditions, ${ }^{8-11}$ but the intermediacy of brookite in titania phase transition is less documented.

Significant progress has been made in the synthetic control of titania nanostructures size, shape and surface morphology. ${ }^{12-17}$ The solvent-thermal method has developed into a versatile tool

${ }^{a}$ Department of Chemistry and Biochemistry, Baylor University, Waco, TX 76796, USA.E-mail: Patrick_Farmer@baylor.edu; Weigang_Lu@baylor. $e d u$

${ }^{b}$ Department of Physics, University of Texas at San Antonio, San Antonio, TX 78249, USA

${ }^{c}$ Electron Microscopy Laboratory, Tulane University, New Orleans, LA 70118, USA

$\uparrow$ Electronic supplementary information (ESI) available: Experimental details concerning the synthesis of VRTNs, characterization of intermediate brookite and anatase nanorods, Fig. S1-S6. See DOI: $10.1039 / \mathrm{c} 2 \mathrm{ce} 06564 \mathrm{a}$ in the production of semiconductor quantum dots (QDs) and other nanostructures. ${ }^{18,19}$ Using this method, several groups have reported the synthesis of titania nanostructures with controlled size, shape and phase. ${ }^{12,14-16,20,21}$ Buonsanti et al. ${ }^{17}$ developed a nonhydrolytic strategy to prepare brookite nanorods with lengths ranging from $30 \mathrm{~nm}$ to $200 \mathrm{~nm}$ using oleic acid/oleylamine as surfactants; initial anatase seeds trigger heterogeneous nucleation of brookite and promote growth of brookite nanorods. In this paper, we present synthesis of V-shaped rutile twinned nanorods (VRTNs) with uniform size and shape via a one-pot high temperature organic method at even higher temperature. Phase changes from anatase and brookite to rutile have been observed.

\section{Results and discussion}

In a typical synthesis, $10 \mathrm{~mL}$ of oleylamine was injected into a three necked flask. $1 \mathrm{~mL}$ of $1 \mathrm{M} \mathrm{TiCl}_{4}$ in toluene was injected into the solvent, and then heated at $180{ }^{\circ} \mathrm{C}$ for 20 minutes to remove toluene and other low boiling point impurities under a vacuum. (Fig. S1† presents ESI mass spectra of different distilled fractions of oleylamine from Acros with an $80-90 \% \mathrm{C}_{18}$ content, the abundance of low boiling point amines after distillation is significantly decreased.) After removal of low boiling point impurities, the temperature was increased to $350{ }^{\circ} \mathrm{C}$ with vigorous stirring. Over the course of reaction, the color of the mixture gradually changed from red to gray, at appropriate time the reaction was quenched by addition of hexane. The product was cooled and precipitated with ethanol and centrifuged and washed several times with a hexane-alcohol solvent. Using this approach, we have successfully produced rutile nanotwins with various diameters and lengths (Fig. 1). The crystal structures of the nanorods were recorded with a Siemens D5000 XRD instrument. Detailed structural investigations were carried out 

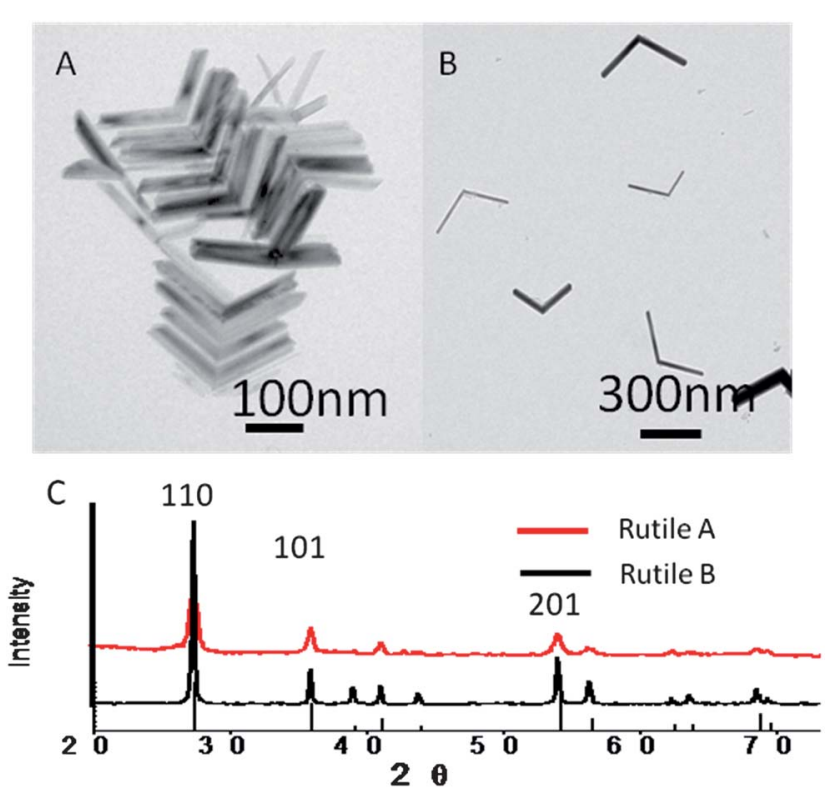

Fig. 1 TEM images and XRD patterns of V-shaped rutile twinned nanorods (VRTNs). (A) VRTNs with arm length $c a .200 \pm 5 \mathrm{~nm}$ (prepared with $5 \mathrm{~mL}$ oleylamine and $1 \mathrm{~mL} 1 \mathrm{M} \mathrm{TiCl}_{4}$ ). (B) VRTNs with arm length $c a .500 \pm 50 \mathrm{~nm}$ (prepared with $10 \mathrm{~mL}$ oleylamine and $1 \mathrm{~mL} 1$ $\mathrm{M} \mathrm{TiCl}_{4}$ ). (C) XRD patterns of two VRTN samples; the red and black patterns were collected from the 200 and $500 \mathrm{~nm}$ arm length samples, respectively. All the peaks can be assigned to the rutile phase. The vertical bar is relative intensity of rutile from JCPDS 21-7216.

using a transmission electron microscope (TEM) and a scanning transmission electron microscope (STEM).

Fig. 1A and $\mathrm{B}$ present typical low resolution TEM images of the VRTNs prepared with different $\mathrm{TiCl}_{4}$ concentrations. The products consist of two kinds of V-shaped nanotwins, straight nanorods and small amounts of multipods in various batches. For a rutile $(h 0 l)[010]$ boundary, the angle of misorientation $\theta$ with respect to the [001] direction in both grains is given by equation $\theta=$ $2 \tan ^{-1}(l a) /(h c)$. For (101) twins, the angle between two twinned bicrystals is $114.5^{\circ}\left(\theta=2 \tan ^{-1}(1 a) /(1 c)\right)$. For (301) twins, the angle between two twinned bicrystals is $54.8^{\circ}\left(\theta=2 \tan ^{-1}(1 a) /(3 c)\right)$. Statistical analysis shows that the (101) twins account for 75 to $82 \%,(301)$ twins are around 12 to $15 \%$ and the straight rods are around 5 to $10 \%$ of products. All the XRD diffraction peaks in Fig. 1C can be assigned to the rutile phase (tetrahedral crystal structure $a=b=4.59 \AA, c=2.96 \AA)$. Compared with the powder XRD pattern (JCPDS 21-7216), the intensity of a (110) peak in the nanorods was significantly enhanced, which suggests that the nanorods grow in the [001] direction, the nanotwin surfaces are dominated by (110) facets which is further confirmed by high resolution transmission electron microscope (HRTEM) images and selected area electron diffraction (SAED) patterns.

Detailed structural information of VRTNs is presented in Fig. 2. Fig. 2A is a low resolution TEM image of a (101) twin with clean surface and perfect symmetry. The angle between two arms of the twins is $\sim 114^{\circ}$; the diameters and aspect ratio of each domain are $42 \mathrm{~nm}$ and 7 , respectively. Selected area electron diffraction (SAED), Fig. 2B-D, of different parts along the twin's structures show that each of the twinned crystals is single crystalline. The SAED from the twin interface in the same

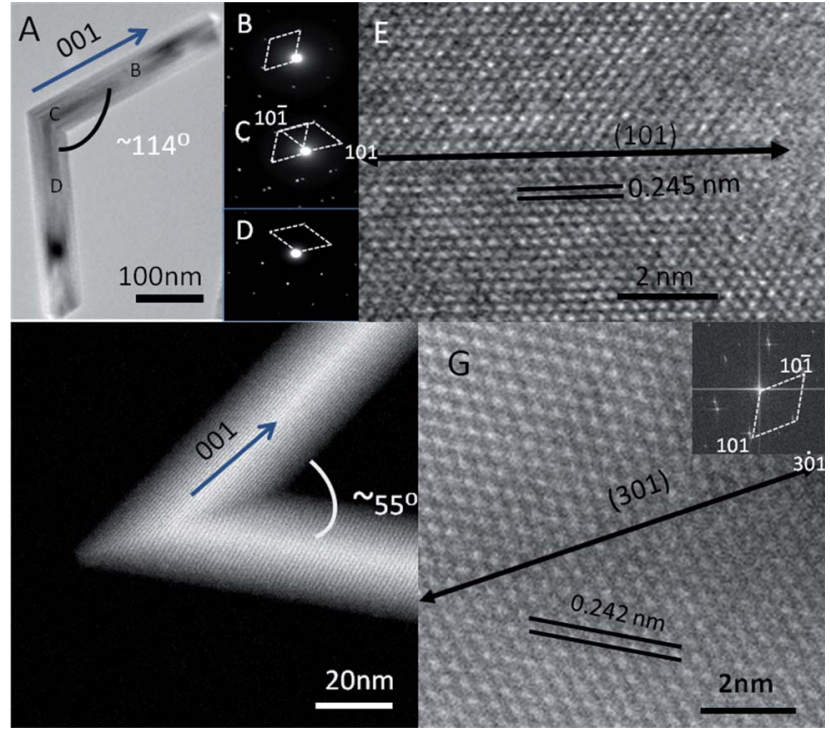

Fig. 2 (A) Low resolution TEM images of (101) rutile twin nanorods, 001 is the growth direction of the nanorods. (B-D) SAED of different parts along (101) twins: (B) up-right arm; (C) twins boundary; (D) lowleft domain. (E) High resolution TEM images of a (101) twins boundary highlighted with a double arrow line. (F) Low resolution HAADFSTEM image of (310) twins. (G) Higher resolution HAADF image of the (310) twins boundary, inset: the FFT pattern shows the (301) plane is the twin boundary.

orientation reveals that both crystals coincide to generate the twin boundary parallel to the (101) plane, Fig. 2D. The surface of the twinned nanorods is smooth and dominated by the (110) surface because the rutile (110) surface has the lowest surface energy. ${ }^{6}$ High resolution TEM images, Fig. 2E, of the interface are consistent with the SAED of the twins interface. The lattice distance between (101) planes is $0.245 \mathrm{~nm}$, which is slightly shorter than that of the bulk rutile $d_{101}=0.248 \mathrm{~nm}$. For (301) twins, each of the domains of the twins is also single crystalline growing in the [001] direction but shares a (301) twins plane, Fig. 2F. The distance between (101) planes is $0.242 \mathrm{~nm}$ also smaller than the bulk counterpart.

The growth of VRTNs was followed by TEM imaging of samples of the reaction solution taken at different time intervals during their formation, Fig. 3. At the beginning of the reaction, there are few rutile twins and many small titania nanorods, Fig. 3A, more and more rutile twins emerged as reaction continued, Fig. 3B, until pure rutile phase formed at 30 minutes, Fig. 3C. XRD patterns of these samples (ESI S2 $\dagger$ ) show two types of diffraction patterns, sharp peaks assigned to the VRTNs and broad peaks due to small titania nanorods. Since XRD patterns of anatase and brookite small nanorods are similar, HRTEM images were taken to determine their phase. The HRTEM images, Fig. 3E and $\mathrm{G}$, and their fast Fourier transform (FFT) patterns, Fig. 3F and $\mathrm{H}$, confirm that the smaller titania nanorods, e.g. in Fig. $3 \mathrm{~B}$, are brookite phase. ${ }^{17}$ As more and more VRTNs emerged, the brookite nanorods transform into smaller nanoparticles with a broader size distribution (ESI S3 $\dagger$ ), which suggests that the brookite nanorods dissolve to support VRTNs growth.

The most important factor in the synthesis of VRTNs is reaction temperature. At $290{ }^{\circ} \mathrm{C}$ only brookite particles are 


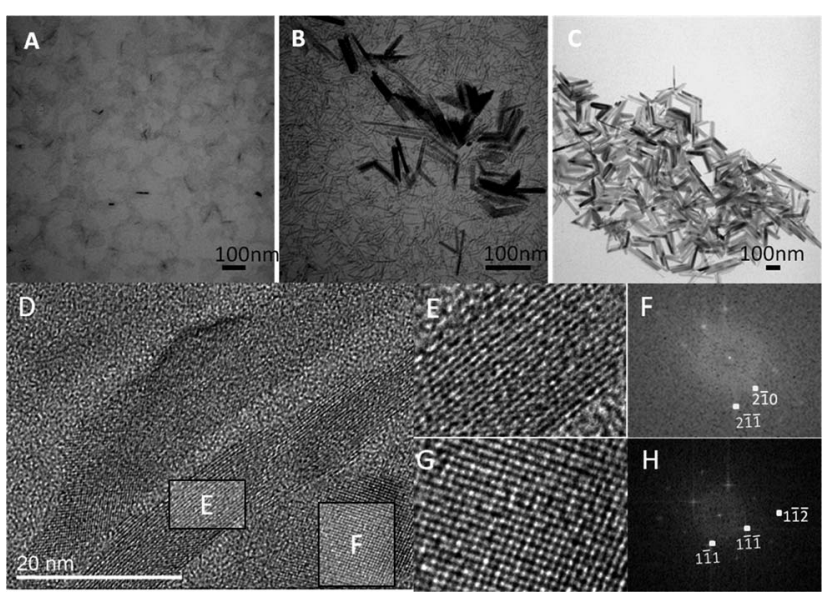

Fig. 3 TEM images at different stages during rutile twins growth. (A) After 2 min, brookite nanorods dominate (light contrast) with a few rutile twins and nanorods present (dark contrast). (B) After 10 min, more rutile nanotwins emerge, and the size distribution of brookite particles widens. (C) After 30 min, essentially pure rutile phase VRTNs are confirmed by XRD and TEM analysis. (D) High resolution TEM images of brookite nanorods from Fig. 2B. (E and F) HRTEM image and FFT pattern of a brookite nanorod viewed along the $\langle 1,2,0\rangle$ zone axis. ( $\mathrm{G}$ and $\mathrm{H}$ ) HRTEM image and FFT pattern of a brookite nanorod viewed along the $\langle 1,1,0\rangle$ zone axis.

observed. ${ }^{17} \mathrm{~A}$ phase change from brookite to rutile is observed at $320^{\circ} \mathrm{C}$ and pure rutile phase is obtained at $350{ }^{\circ} \mathrm{C}$. Thus reaction temperature is essential for the brookite-to-rutile phase change that enables VRTN formation.

The perfect symmetry of the VRTN domains and their smooth surfaces observed at different growth stages implies that the rutile nanotwins are formed at the nucleation stage instead of by oriented attachment. ${ }^{22,23}$ The monomer concentration, as well as external and internal environmental factors must favor the formation of twin crystals. In other words, if under certain conditions two titania monomers cluster to form a low energy combination displaced relative to the normal lattice position, it forms a VRTN seed which persists and grows. ${ }^{24}$ Thus the titania monomer concentration at high temperature will be an important factor in the VRTNs growth. In transformation from brookite to rutile phase, the monomer concentration is determined by brookite nanorod's dissolution rate. We hypothesized that if more than two monomers or clusters coalescence at the same time, titania multipods should form. As a test of this hypothesis, a higher monomer concentration was achieved by rapid addition of $1 \mathrm{~mL}$ of $1 \mathrm{M}$ titania precursor at $200{ }^{\circ} \mathrm{C}$ in oleylamine into a oleylamine solution at $360{ }^{\circ} \mathrm{C}$; TEM imaging of the resulting product solution did indeed show a high percentage of rutile multipods and dendrimers produced (Fig. 4A).

If the reaction is carried out with no stirring at $290{ }^{\circ} \mathrm{C}$, the growth of anatase and brookite nanorods is observed. As shown in Fig. 4B and $\mathrm{C}$, the nanorods with smaller contrast are brookite; the nanorods with larger contrasts are anatase. The rough surface of the anatase nanorods generated under these conditions suggests that they grow through oriented attachment, Fig. 4C. The Brownian movement of anatase seeds in the unstirred reaction solution allows their active planes to align and attach to form oriented anatase nanorods. The heterogeneous

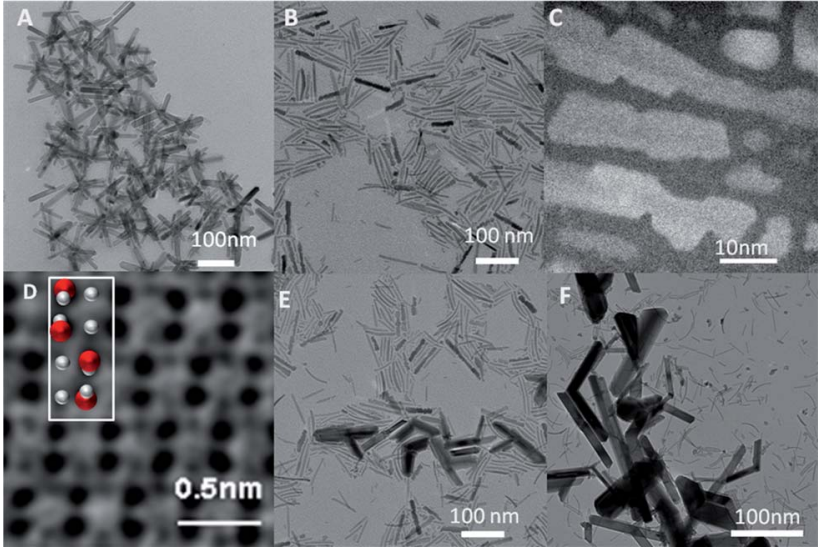

Fig. 4 (A) TEM image of rutile multipods and dendrimers synthesized by rapidly injecting the titania precursor into $360^{\circ} \mathrm{C}$ oleylamine (B) TEM image of coexisting brookite and anatase nanorods synthesized at $290^{\circ} \mathrm{C}$. (C) STEM image of anatase nanorods shows features of oriented attachment. (D) Atomic resolution bright field STEM image of the anatase (100) surface, the larger black spots showing Ti rows; the smaller black features are $\mathrm{O}$ atoms; the inset model superimposes the anatase unit cell. (E) The emergence of rutile nanotwins after 5 minutes at $350{ }^{\circ} \mathrm{C}$ without stirring. (F) Brookite and anatase nanorods dissolved after 20 minutes at $350^{\circ} \mathrm{C}$ support growth of VRTNs.

nucleation of brookite onto anatase seeds is suppressed due to the decreased number of anatase seeds. This effect has also been observed in the synthesis of polyaniline nanofibers ${ }^{25}$ and silica nanoparticles. ${ }^{26}$ Both brookite and anatase nanorods may then dissolve into monomers leading to the growth of VRTNs at $350{ }^{\circ} \mathrm{C}$, Fig. 4E and F, which occurs in the absence of stirring.

Based on the above observations, we propose dual pathways for formation of VRTNs, dependent on experimental conditions. With stirring, brookite nanorods form through a self-regulated seed-catalyzed mechanism, step I in Fig. 5. In this pathway, anatase seeds trigger the heterogeneous nucleation of brookite and promote subsequent growth of the brookite nanorods (ESI S5 $†$ ). ${ }^{17}$ Without stirring, anatase seeds grow into anatase nanorods through oriented attachment (step III) as well as through formation of brookite nanorods. The brookite-to-rutile

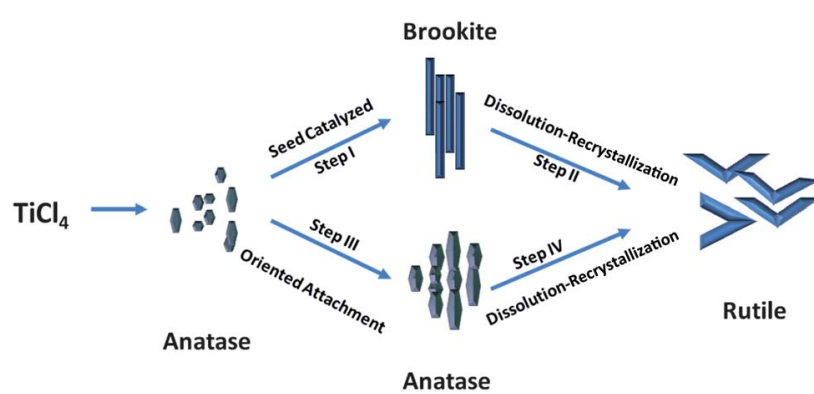

Fig. 5 Proposed two-path mechanism for the formation of rutile nanotwins. (Step I) Anatase seeds catalyzed the anatase-to-brookite phase transition during brookite nanorods growth. (Step II) Dissolution of the brookite nanorods accompanies the formation of rutile nanotwins. (Step III) Anatase nanorods are formed through oriented attachment. (Step IV) Dissolution of anatase nanorods allows for the formation of rutile nanotwins. 
(step II) and anatase-to-rutile (step IV) conversions occur when the temperature is high enough to overcome the inherent energy barrier to form the rutile phase.

Much evidence supports this hypothesis. The emergence of the VRTNs is accompanied by dissolution of brookite and anatase nanorods. The broadened size distribution of brookite and anatase nanorods suggests that the formation of VRTNs takes place through a dissolution-recrystallization process from both types of nanorods. Thus, a temperature dependent dissolution of both brookite and anatase nanorods seems to provide the needed titania to support the rutile nanotwins growth.

\section{Conclusions}

In summary, we have demonstrated a large scale synthesis of VRTNs using a one-pot high temperature solvothermal method with oleylamine serving as solvent and capping ligand. Using this method, high quality VRTNs with exposed (110) facets are produced. A dual pathways mechanism is proposed for the formation of rutile nanotwins through both anatase and brookite nanoparticles. The availability of rutile nanotwins provides an ideal material to study atomic structures of rutile twins with genuine atomic resolution using analytical STEM. This methodology may fill the gap between surface science and practical catalytic applications.

\section{Experimental}

\section{Materials}

Titanium chloride (99.9\% Aldrich), oleylamine (Acros), anhydrous hexane (VWR), anhydrous toluene (VWR) and anhydrous ethanol (VWR) were used as received without further purification. A stock $1 \mathrm{M} \mathrm{TiCl}{ }_{4}$ solution was prepared by injecting $5.46 \mathrm{~mL} \mathrm{TiCl}_{4}$ into $50 \mathrm{~mL}$ anhydrous toluene.

\section{Characterizations}

The X-ray diffraction patterns were recorded on a Siemens D5000 XRD instrument. Multiple instruments including JEOL 1100, JEOL 2010F and probe corrected JEOL ARM 200F were employed for the EM investigations.

\section{Synthesis of V-shaped rutile nanotwins, VRNTs}

In a typical synthesis of rutile nanotwins, a $1 \mathrm{~mL}$ sample of the stock $\mathrm{TiCl}_{4}$ solution was injected into $10 \mathrm{~mL}$ oleylamine, then heated to $180{ }^{\circ} \mathrm{C}$ for 20 minutes to remove toluene and other low boiling impurities under a vacuum. The temperature was then increased to $350{ }^{\circ} \mathrm{C}$ with vigorous stirring. During this time, the color of the reaction mixture changed from red to gray over $15 \mathrm{~min}$. The reaction was quenched by adding room temperature hexane into the flask, and solid products were isolated by adding a sufficient amount of ethanol and separating by centrifugation. The solid VRNTs were washed several times with a hexanealcohol solvent mixture before characterization.

\section{Synthesis of brookite nanorods}

To generate brookite nanorods, a $1 \mathrm{~mL}$ sample of the $1 \mathrm{M} \mathrm{TiCl}_{4}$ stock solution was injected into $10 \mathrm{~mL}$ oleylamine and heated to
$180{ }^{\circ} \mathrm{C}$ for 20 minutes to remove toluene and other impurities under a vacuum. The temperature was increased to $290{ }^{\circ} \mathrm{C}$ with vigorous stirring for $20 \mathrm{~min}$ before quenching with hexane. The solid brookite nanorods were isolated and washed as mentioned above in the synthesis of VRNTs. In the absence of stirring, this procedure produced a mixture of brookite and anatase nanorods, as described in the text.

\section{Synthesis of rutile multipods}

A $10 \mathrm{~mL}$ sample of oleylamine was heated to $180{ }^{\circ} \mathrm{C}$ for 20 minutes to remove low boiling impurities under a vacuum in a three neck flask; the sample temperature was then increased to $360{ }^{\circ} \mathrm{C}$. A $1 \mathrm{~mL}$ sample of $1 \mathrm{M} \mathrm{TiCl}_{4}$ in oleylamine at $200{ }^{\circ} \mathrm{C}$ was rapidly injected into the $360^{\circ} \mathrm{C}$ oleylamine solution. The reaction temperature decreased to $335^{\circ} \mathrm{C}$ shortly before rising again to $360{ }^{\circ} \mathrm{C}$. The reaction mixture was quenched with hexane, separated and washed as mentioned above.

\section{Acknowledgements}

The authors acknowledge funding and support from Baylor University (PJF) and the NSF PREM grant \# DMR 0934218 (MJY), Title: Oxide and metal nanoparticles - the interface between life sciences and physical sciences.

\section{Notes and references}

1 M. Gratzel, Nature, 2001, 414, 338-344, DOI: 10.1038/35104607.

2 A. Fujishima and K. Honda, Nature, 1972, 238, 37, DOI: 10.1038/ $238037 \mathrm{a} 0$.

3 B. Oregan and M. Gratzel, Nature, 1991, 353, 737-740, DOI: 10.1038/ $353737 \mathrm{a} 0$.

4 Z. Zhang, R. Rousseau, J. Gong, B. D. Kay and Z. Dohnalek, J. Am. Chem. Soc., 2009, 131, 17926-17932, DOI: 10.1021/ ja907431s.

5 Z. Zhang, R. Rousseau, J. Gong, S. Li, B. D. Kay, Q. Ge and Z. Dohnalek, Phys. Rev. Lett., 2008, 101, 156103, DOI: 10.1103/ PhysRevLett.101.156103.

6 U. Diebold, Surf. Sci. Rep., 2003, 48, 53-229, DOI: 10.1016/S01675729(02)00100-0.

7 N. Daneu, H. Schmid, A. Recnik and W. Mader, Am. Mineral., 2007, 92, 1789-1799, DOI: 10.2138/am.2007.2634.

8 X. Chen and S. S. Mao, Chem. Rev., 2007, 107, 2891-2959, DOI: $10.1021 / \mathrm{cr} 0500535$.

9 K. Ding, Z. Miao, B. Hu, G. An, Z. Sun, B. Han and Z. Liu, Langmuir, 2010, 26, 10294-10302, DOI: 10.1021/la100468e.

10 Y. Jun, J. Choi and J. Cheon, Angew. Chem., Int. Ed., 2006, 45, 3414 3439, DOI: 10.1002/anie.200503821.

11 M. Niederberger, Acc. Chem. Res., 2007, 40, 793-800, DOI: 10.1021/ $\operatorname{ar} 600035 \mathrm{e}$.

12 A. Chemseddine and T. Moritz, Eur. J. Inorg. Chem., 1999, 235-245, DOI: 10.1002/(SICI)1099-0682(19990202)1999:2<235:AID-EJIC235> 3.0. $\mathrm{CO} ; 2-\mathrm{N}$.

13 R. Buonsanti, V. Grillo, E. Carlino, C. Giannini, F. Gozzo, M. Garcia-Hernandez, M. A. Garcia, R. Cingolani and P. D. Cozzoli, J. Am. Chem. Soc., 2010, 132, 2437-2464, DOI: $10.1021 / j a 910322 \mathrm{a}$.

14 K. Ding, Z. Miao, B. Hu, G. An, Z. Sun, B. Han and Z. Liu, Langmuir, 2010, 26, 5129-5134, DOI: 10.1021/la903600q.

15 P. D. Cozzoli, A. Kornowski and H. Weller, J. Am. Chem. Soc., 2003, 125, 14539-14548, DOI: $10.1021 / \mathrm{ja} 036505 \mathrm{~h}$.

16 A. Kandiel, R. Dillert, A. Feldhoff and D. Bahnemann, J. Phys. Chem. C, 2010, 114, 4909-4915, DOI: 10.1021/jp912008k.

17 R. Buonsanti, V. Grillo, E. Carlino, C. Giannini, T. Kipp, R. Cingolani and P. D. Cozzoli, J. Am. Chem. Soc., 2008, 130, 11223-11233, DOI: 10.1021/ja803559b. 
18 C. B. Murray, D. J. Norris and M. G. Bawendi, J. Am. Chem. Soc., 1993, 115, 8706-8715, DOI: 10.1021/ja00072a025.

19 X. G. Peng, L. Manna, W. D. Yang, J. Wickham, E. Scher, A. Kadavanich and A. P. Alivisatos, Nature, 2000, 404, 59-61, DOI: $10.1038 / 35003535$.

20 M. Niederberger, M. H. Bartl and G. D. Stucky, J. Am. Chem. Soc., 2002, 124, 13642-13643, DOI: 10.1021/ja027115i.

21 B. Wu, C. Guo, N. Zheng, Z. Xie and G. D. Stucky, J. Am. Chem. Soc., 2008, 130, 17563-17567, DOI: 10.1021/ja8069715.
22 W. Lu, P. Gao, W. Bin Jian, Z. Wang and J. Fang, J. Am. Chem. Soc., 2004, 126, 14816-14821, DOI: 10.1021/ja046769j.

23 W. Koh, A. C. Bartnik, F. W. Wise and C. B. Murray, J. Am. Chem. Soc., 2010, 132, 3909-3913, DOI: 10.1021/ja9105682.

24 M. J. Buerger, Am. Mineral., 1945, 30, 469-482.

25 D. Li and R. B. Kaner, J. Am. Chem. Soc., 2006, 128, 968-975, DOI: $10.1021 / \mathrm{ja} 056609 \mathrm{n}$.

26 W. Stober, A. Fink and E. Bohn, J. Colloid Interface Sci., 1968, 26, 62, DOI: 10.1016/0021-9797(68)90272-5. 